\title{
Research of the Transformer Fault Diagnosis Expert System based on ESTA and Deep Learning Neural Network programmed in MATLAB
}

\author{
Qiming Cui ${ }^{1, a}$, Kai Liang ${ }^{1, b}$,Hongyu Gao ${ }^{2, \mathrm{c}}$, Guodong Chen ${ }^{2, \mathrm{~d}}$,Naiyu \\ Wang $^{2, \mathrm{e}}$,Shaoyu Jin ${ }^{1, \mathrm{f}}$,Changqing Zhong ${ }^{1, \mathrm{~g}}$,Shuting Cui ${ }^{3, \mathrm{~h}}$, Daojun Sun ${ }^{1, \mathrm{i}}, \mathrm{Jie}$ \\ Fang ${ }^{4, j}$,Chengwei Han ${ }^{1, k}$, Bin X $u^{1, l}$, Yuhong $\mathrm{An}^{5, \mathrm{~m}}$, ,Minghu $\mathrm{Xu}^{6, \mathrm{n}}$ \\ ${ }^{1}$ State Grid Liaoning Anshan Power Supply Company,Anshan 114001,China \\ ${ }^{2}$ State Grid of China Technology College, Taian 271000,China \\ ${ }^{3}$ Eastern Michigan University, Ypsilanti,MI 48197,the U.S.A. \\ ${ }^{4}$ State Grid Weinan Power Supply Company, Weinan 714200, China \\ ${ }^{5}$ State Grid Liaoning Dalian Training Center, Dalian 116023,China \\ ${ }^{6}$ State Grid Liaoning Electric Power Supply Skills Training Center, Jinzhou 121001, China \\ aospb2002@aliyun.com, ${ }^{b} 13009376153 @$ sina.com, ${ }^{\circ}$ gaohongyu098@sina.com, ${ }^{\mathrm{d}} \mathrm{cgdta} @ 126 . c o m$, \\ esdtawny@163.com, ${ }^{\mathrm{f}} 707897891 @ q q . c o m,{ }^{\mathrm{g}} 309074907 @ q q . c o m,{ }^{\mathrm{h}}$ dreamilk@126.com, ${ }^{1} 1991393560$ \\ @qq.com,'bpqbfq.2010@qq.com, ${ }^{\text {kh } h s w 2014 @ s i n a . c n, ~ ' f c y 500 @ q q . c o m, ~}{ }^{\mathrm{m}} 284813744 @ q q . c o m$, \\ nxuminghuok@163.com
}

Keywords: Expert System; ESTA; Deep Belief Network; BP Neural Network; MATLAB; Transformer Fault Diagnosis

Abstract. Some procedures or functions had be added to an ESTA (Expert System Shell for Text Animation) so that the ESTA and MATLAB can communicate via some data files.On this basis,a deep learning-DBN(Deep Belief Network) and two BP(back propagation) artificial neural network based on the MATLAB programming were researched by using directly DGA (Dissolved Gas Analysis) and characteristic gas method in transformer oil chromatographic analysis. The transformer fault diagnosis expert system based on a three ratio and characteristic gas method of DGA and ESTA including the DBN and two BP artificial neural network programmed in MATLAB had be created.The basic application shows the effectiveness of the expert system.

\section{Introduction}

ANN (Artificial Neural Network) including BP, because of its inherent strong adaptation and learning ability, has been applied very widely in many fields of automatic control, fault diagnosis and detection[1]. MATLAB is a high performance numerical computing and visualization software, its programming language is called the fourth generation computer language and is more readable, debugging simple[1]. In 2006, Hinton et al. put forward the concept of deep learning. It is a very close to the artificial intelligence in machine learning field, the motivation is to establish and simulate the neural networks of human brain analysis and learning.Method of using stack RBM (Restricted Boltzmann Machines) constituting a deep neural network is known as DBN (Deep Belief Network) in deep learning.In general,a DBN can be composed of a number of layer RBM and a layer of BP network [2], it is a kind of generation model. By training the weights between the neurons, it can let whole neural network according to the maximum probability to generate training data,then the DBN can identify and classify data.An expert system is an intelligent computer program, it can solve some difficult problems by using some knowledges of experts and reasoning processes[3].Transformer fault diagnosis generally can identify some fault components by measuring and analyzing some change information related to gas content in transformer oil.This paper discusses the application of transformer internal fault diagnosis by using an expert system based on a three ratio,characteristic gas method in DGA and ESTA combined with the DBN and BP artificial neural networks programmed in MATLAB. 
Three ratio and characteristic gas method in Transformer DGA. IEEE believes that the most effective method of transformer internal-fault-early-diagnosis is dissolved gas analysis or oil chromatographic analysis in transformer oil[4]. For early fault detection of a transformer, it is accurate and continuous, and it can determine some faults that is difficult to find by using other methods. According to the dissolved gases $\left(\mathrm{H}_{2}, \mathrm{CH}_{4}, \mathrm{C}_{2} \mathrm{H}_{6}, \mathrm{C}_{2} \mathrm{H}_{4}, \mathrm{C}_{2} \mathrm{H}_{2}, \mathrm{CO}, \mathrm{CO}_{2}\right)$ content and its trend of development in transformer oil, some preliminary analysis can be done by the three ratio and characteristic gas method to detect transformer internal latent fault. The fault types determining standards of the three ratio and characteristic gas method are given below[5], see Table1 and Table2. Basic characteristics of the ESTA and the Expert System based on ESTA. The ESTA supports mixed deterministic reasoning, its knowledge base consists of some sections, parameters and rules etc.[6]. The ESTA can search a state space of forward chaining and/or backward chaining (text symbols).In a section, the ESTA can set up or abandon some parameters through backward chaining and also can move from one section to another so as to realize the forward chaining in the state space searching.In the research expert system, the knowledge base uses forward/backward chaining mixed reasoning and includes determining of the three ratio,characteristic gas method,and training/prediction modules of the $\mathrm{DBN}$ and two $\mathrm{BP}$ neural network based on MATLAB programming. For example, following rules implementing the three ratio method are used in the $\operatorname{ESTA}($ partly corresponding to the Table 1$)$ :

if $\left(\mathrm{C}_{2} \mathrm{H}_{2} / \mathrm{C}_{2} \mathrm{H}_{4}<0.1\right)$ (assign $\left.\mathrm{C}_{2} \mathrm{H}_{2} \mathrm{C}_{2} \mathrm{H}_{4}:=0\right) \quad$; The $\mathrm{C}_{2} \mathrm{H}_{2}, \mathrm{C}_{2} \mathrm{H}_{4}$ and $\mathrm{C}_{2} \mathrm{H}_{2} \mathrm{C}_{2} \mathrm{H}_{4}$ are variables. if $\left(\mathrm{CH}_{4} / \mathrm{H}_{2}>=1\right.$ and $\left.\mathrm{CH}_{4} / \mathrm{H}_{2}<3\right)\left(\right.$ assign $\left.\mathrm{CH}_{4} \mathrm{H}_{2}:=2\right)$; The assign is assignment statement. if $\left(\mathrm{C}_{2} \mathrm{H}_{4} / \mathrm{C}_{2} \mathrm{H}_{6}>=1\right.$ and $\left.\mathrm{C}_{2} \mathrm{H}_{4} / \mathrm{C}_{2} \mathrm{H}_{6}<3\right)$ (assign $\mathrm{C}_{2} \mathrm{H}_{4} \mathrm{C}_{2} \mathrm{H}_{6}:=1$ )

Table 1 encoding rules of the three ratio method and fault types diagnosis

\begin{tabular}{|c|c|c|c|}
\hline $\mathrm{C}_{2} \mathrm{H}_{2} / \mathrm{C}_{2} \mathrm{H}_{4}$ & $\mathrm{CH}_{4} / \mathrm{H}_{2}$ & $\mathrm{C}_{2} \mathrm{H}_{4} / \mathrm{C}_{2} \mathrm{H}_{6}$ & Gas ratio range/Fault types diagnosis \\
\hline 0 & 1 & 0 & $<0.1$ \\
\hline 1 & 0 & 0 & $\geq 0.1 \sim<1$ \\
\hline 1 & 2 & 1 & $\geq 1 \sim<3$ \\
\hline 2 & 2 & 2 & $\geq 3$ \\
\hline \multirow{5}{*}{0} & 1 & 0 & local discharge \\
\hline & 0 & 1 & low temperature overheat (under $150 \square$ ) \\
\hline & 2 & 0 & low temperature overheat $(150 \square-300 \square)$ \\
\hline & 2 & 1 & medium temperature overheat $(300 \square-700 \square)$ \\
\hline & $0,1,2$ & 2 & high temperature overheat (above 700 $\square$ ) \\
\hline \multirow{2}{*}{2} & 0,1 & $0,1,2$ & low energy (spark) discharge \\
\hline & 2 & $0,1,2$ & low energy (spark) discharge and overheat \\
\hline \multirow{2}{*}{1} & 0,1 & $0,1,2$ & arc discharge \\
\hline & 2 & $0,1,2$ & arc discharge and overheat \\
\hline
\end{tabular}

Table 2 characteristics gas method and fault natures

\begin{tabular}{|l|l|}
\hline \multicolumn{1}{|c|}{ Characteristics gas } & \multicolumn{1}{|c|}{ Fault natures } \\
\hline total hydrocarbon is relatively high, $\mathrm{C}_{2} \mathrm{H}_{2}<5^{*} 10^{-6}$ & general overheat fault \\
\hline $\begin{array}{l}\text { total hydrocarbon is high, } \mathrm{C}_{2} \mathrm{H}_{2}>5^{*} 10^{-6}, \mathrm{C}_{2} \mathrm{H}_{2} \text { didn't constitute } \\
\text { main component of total hydrocarbon,content of } \mathrm{H}_{2} \text { is high }\end{array}$ & serious overheat fault \\
\hline $\begin{array}{l}\text { total hydrocarbon is not high, } \mathrm{H}_{2}>10^{*} 10^{-6}, \mathrm{CH}_{4} \text { is the main } \\
\text { component of total hydrocarbon }\end{array}$ & local discharge \\
\hline $\begin{array}{l}\text { total hydrocarbon is high, } \mathrm{C}_{2} \mathrm{H}_{2}>100^{*} 10^{-6}, \text { content of } \mathrm{H}_{2} \text { is } \\
\text { relatively high }\end{array}$ & spark discharge \\
\hline $\begin{array}{l}\text { total hydrocarbon is high, } \mathrm{C}_{2} \mathrm{H}_{2} \text { is high and it constitute the } \\
\text { main component of total hydrocarbon,content of } \mathrm{H}_{2} \text { is high }\end{array}$ & arc discharge \\
\hline
\end{tabular}

Transformer fault diagnosis based on two BP neural network in MATLAB programming. This section describes transformer fault diagnosis based on BP neural network programmed in MATLAB. 
The first BP neural network. BP artificial neural network has good function of pattern classification and is suitable for fault diagnosis problem. It is composed of some input nodes, hidden and output. Two BP artificial neural network called in the research expert system are realized by programming in the MATLAB. For example, some correction MATLAB codes of computing neural network weights and threshold is as follows[7]:

for $\mathrm{i}=1: 1$ :midnum $\mathrm{S}=1 /(1+\exp (-\mathrm{I}(\mathrm{i})))$; $\mathrm{FI}(\mathrm{i})=\mathrm{S}^{*}(1-\mathrm{S})$; end

for $\mathrm{k}=1: 1$ :innum for $\mathrm{j}=1: 1$ :midnum esum $=0$; for $\mathrm{o}=1: 1$ :outnum esum $=\mathrm{esum}+\mathrm{e}(\mathrm{o})^{*} \mathrm{w} 2(\mathrm{j}, \mathrm{o})$; end

$\mathrm{dw}(\mathrm{k}, \mathrm{j})=\mathrm{FI}(\mathrm{j}) * \mathrm{x}(\mathrm{k}) *$ esum; $\mathrm{db}(\mathrm{j})=\mathrm{FI}(\mathrm{j}) *$ esum; end end.

The first BP artificial neural network uses $\mathrm{H}_{2}, \mathrm{CH}_{4}, \mathrm{C}_{2} \mathrm{H}_{6}, \mathrm{C}_{2} \mathrm{H}_{4}, \mathrm{C}_{2} \mathrm{H}_{2}, \mathrm{CO}, \mathrm{CO}_{2}$ values directly as input of it[8], two types of fault (overheat and arc discharge) as its output, and a layer of hidden nodes(30 neurons).

The second BP neural network. The second BP artificial neural network uses the characteristic gas method(see Table2). According to the $\mathrm{X}_{1}$ represents $\mathrm{H}_{2}, \mathrm{X}_{2}$ represents $\mathrm{C}_{1}+\mathrm{C}_{2}$ (total hydrocarbon), $\mathrm{X}_{3}$ represents $\mathrm{C}_{2} \mathrm{H}_{2}, \mathrm{X}_{4}$ represents $\mathrm{C}_{2} \mathrm{H}_{2} / \mathrm{X}_{2}, \mathrm{X}_{5}$ represents $\mathrm{CH}_{4} / \mathrm{X}_{2}, \mathrm{C}_{1}+\mathrm{C}_{2}$ is sum of $\mathrm{CH}_{4}, \mathrm{C}_{2} \mathrm{H}_{6}, \mathrm{C}_{2} \mathrm{H}_{4}$ and $\mathrm{C}_{2} \mathrm{H}_{2}$ values, then membership function can be defined as follows[9]:

$$
\mathbf{X}_{1}, \mathbf{X}_{2}=\left[\begin{array}{l}
0.005 \mathrm{X} ; \mathrm{X} \in(0,100) \\
0.01 \mathrm{X}-0.5 ; \mathrm{X} \in(100,150) \\
1 ; \mathrm{X} \geq 150
\end{array}\right] ; \mathbf{X}_{3}=\left[\begin{array}{l}
0.1 \mathrm{X} ; \mathrm{X} \in(0,10) \\
1 ; \mathrm{X} \geq 10
\end{array}\right]
$$

Some rules of the expert system are determined by the functions. After $\mathrm{H}_{2}, \mathrm{CH}_{4}, \mathrm{C}_{2} \mathrm{H}_{6}, \mathrm{C}_{2} \mathrm{H}_{4}$ and $\mathrm{C}_{2} \mathrm{H}_{2}$ values are fuzzy processed, the $\mathrm{X}_{1}, \mathrm{X}_{2}, \mathrm{X}_{3}, \mathrm{X}_{4}$ and $\mathrm{X}_{5}$ become five input of the second $\mathrm{BP}$ artificial neural network, the output is 5 nodes (corresponding to the fault natures of Table 2), a hidden layer nodes (12 neurons). For example, the rule of processing $\mathrm{X}_{1}$ is as follows:

if $\left(\mathrm{H}_{2}>=0\right.$ and $\left.\mathrm{H}_{2}<100\right)$ (assign $\left.\mathrm{X}_{1}:=0.005 * \mathrm{H}_{2}\right)$

if $\left(\mathrm{H}_{2}>=100\right.$ and $\left.\mathrm{H}_{2}<150\right)$ (assign $\left.\mathrm{X}_{1}:=0.01 * \mathrm{H}_{2}-0.5\right)$

if $\left(\mathrm{H}_{2}>=150\right)\left(\right.$ assign $\left.\mathrm{X}_{1}:=1\right)$.

Transformer fault diagnosis based on the DBN in MATLAB programming. This paper builds a DBN. It consists of six RBM stack layer and a BP layer,and a classifier at the top is softmax(see Fig.1).Input data of the DBN training includes the values of $\mathrm{H}_{2}, \mathrm{CO}, \mathrm{CH}_{4}, \mathrm{C}_{2} \mathrm{H}_{4}, \mathrm{C}_{2} \mathrm{H}_{2}, \mathrm{C}_{2} \mathrm{H}_{6}$ (coming from a transformer gas monitoring system).Output is classified as normal (corresponding output coding is 001), arc discharge (100), overheat(010). Training data is 150 records, and training with label is 2000 times. Input data of the DBN testing comes from the 16 rows and the $\mathrm{H}_{2}, \mathrm{CO}, \mathrm{CH}_{4}$, $\mathrm{C}_{2} \mathrm{H}_{4}, \mathrm{C}_{2} \mathrm{H}_{2}, \mathrm{C}_{2} \mathrm{H}_{6}$ columns in Table 3. A calculation results as shown in Fig.2, correct ratio: 0.8125.

The partly program codes of the DBN is as follows:

Xmean $=$ mean $(X) ; \quad$ Calculating the average value of the input variables.

$X s t d=\operatorname{std}(X) ; \quad$ Calculating the variance of the input variables.

$\mathrm{X}=$ bsxfun( @ rdivide,bsxfun(@minus,X,Xmean),Xstd); Initializing the input variables.

$\operatorname{rbm} 1=\operatorname{rbm}([6,100]) ; \quad$ Creating the first layer RBM, RBM training gets the first hidden layer of network parameters.

rbm1 = checkrbmtrain(@rbmtrain,rbm1,X,50,0.1); @ rbmtrain show that data is processed in accord with the binomial distribution. The rbm1 input data is $\mathrm{X}$,the fourth parameter checks whether or not stop training every 50 times, the fifth parameter indicates the initial learning efficiency. net_rbm1 $=\operatorname{rbm} 2$ nnet $\left(\operatorname{rbm} 1\right.$, 'up'$\left.^{\prime}\right)$; The RBM is converted to a neural network for subsequent processing.

$\mathrm{h} 1=$ nnetfw(net_rbm1,X); $\quad$ The result of the RBM transformation is used to train the next layer. Other layers RBM is omitted. 


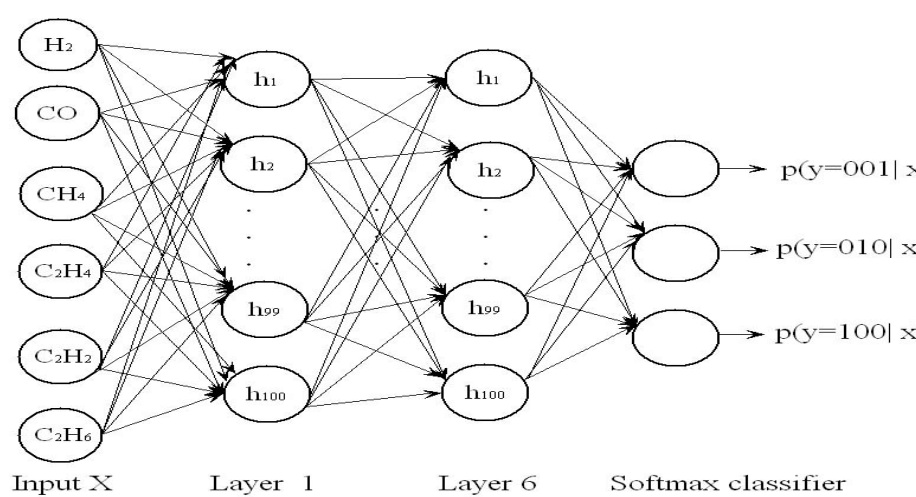

Fig. 1 the schematic diagram of the DBN net $1=\operatorname{nnet}([6,100,200,300,200,100,100,3]$,'softmax'); Multilayer neural network is created, and the result of RBM initialization is configured to the network.

net1.w $\{1\}=$ net_rbm1.w $\{1\} ; \quad$ The first $\mathrm{W}$ (weight) of the net1 comes from the rbm1.

Other is omitted.

net2 = nnettrain(net1,X,Y,2000); Training with label is 2000 times, the BP training is carried out on the deep learning network.

$\mathrm{Y} 2=\mathrm{nnetfw}($ net2,X1); $\quad$ The classification is carried out by using trained depth network net2, the $\mathrm{X} 1$ is test input data, the $\mathrm{Y} 2$ is the test output data.

$\mathrm{Y} 3=$ nnettest(net2,X1,Y1); The $\mathrm{X} 1$ is test input data, the $\mathrm{Y} 1$ is expected output, the $\mathrm{Y} 3$ is correct ratio.

Table 3 the test data,practical conclusion, and the DBN classifier codes

\begin{tabular}{|r|r|r|r|r|r|r|l|l|c|}
\hline $\begin{array}{l}\text { Data } \\
\text { No. }\end{array}$ & \multicolumn{1}{c|}{$\mathrm{H}_{2}$} & $\mathrm{CO}$ & $\mathrm{CH}_{4}$ & $\mathrm{C}_{2} \mathrm{H}_{4}$ & $\mathrm{C}_{2} \mathrm{H}_{2}$ & $\mathrm{C}_{2} \mathrm{H}_{6}$ & $\mathrm{CO}_{2}$ & $\begin{array}{l}\text { Practical } \\
\text { conclusion }\end{array}$ & Codes \\
\hline 1 & 98 & 13 & 252 & 646 & 22 & 95 & 1111 & arc discharge & 100 \\
\hline 2 & 679 & 9 & 4992 & 3671 & 0 & 1873 & 1235 & overheat & 010 \\
\hline 3 & 42 & 20 & 62 & 63 & 73 & 5 & 2917 & arc discharge & 100 \\
\hline 4 & 557 & 0 & 3541 & 2928 & 7 & 521 & 1869 & overheat & 010 \\
\hline 5 & 240 & 0 & 28 & 26 & 85 & 6 & 10 & overheat & 010 \\
\hline 6 & 851 & 17 & 2802 & 3521 & 10 & 772 & 1206 & overheat & 010 \\
\hline 7 & 10 & 39 & 4 & 33 & 6 & 3 & 2907 & overheat & 010 \\
\hline 8 & 100 & 551 & 170 & 34 & 0 & 595 & 4027 & overheat & 010 \\
\hline 9 & 403 & 150 & 1768 & 1302 & 2 & 200 & 10 & overheat & 010 \\
\hline 10 & 97 & 152 & 405 & 583 & 34 & 190 & 2294 & arc discharge & 100 \\
\hline 11 & 68.59 & 266.17 & 24.45 & 0.88 & 0 & 1.95 & & normal & 001 \\
\hline 12 & 67.72 & 253.51 & 24.09 & 0.85 & 0 & 1.88 & & normal & 001 \\
\hline 13 & 66.55 & 243.84 & 23.87 & 0.81 & 0 & 1.96 & & normal & 001 \\
\hline 13 & 72.5 & 287.06 & 25 & 0.92 & 0 & 1.93 & & normal & 001 \\
\hline 15 & 71.84 & 277.43 & 24.82 & 0.91 & 0 & 2.01 & & normal & 001 \\
\hline 16 & 9.66 & 402.1 & 2.94 & 0 & 0 & 0.26 & & normal & 001 \\
\hline
\end{tabular}

The Expert System combined with the DBN and BP neural network in MATLAB. The DBN module realized by MATLAB can be called in the transformer fault diagnosis expert system realized by ESTA after it is compiled. Training and testing data are given in the form of operation system file in the DBN module.

The two BP artificial neural network model will be called in the research expert system after MATLIB compiling it and generating some executable programs. When the neural networks is trained, sample data are preprocessed by using rules in the knowledges base such that some meaningless sample data (such as not conforming to the specified value or the extreme data) can be ruled out. After the neural networks is trained, some weights and other parameters as data files will be stored for later using whenever diagnosis or prediction (see Fig.3). When diagnosing, the values of $\mathrm{H}_{2}, \mathrm{CH}_{4}, \mathrm{C}_{2} \mathrm{H}_{6}, \mathrm{C}_{2} \mathrm{H}_{4}, \mathrm{C}_{2} \mathrm{H}_{2}, \mathrm{CO}, \mathrm{CO}_{2}$ are inputted and also preprocessed. The expert system does 
reasoning by calling relation rules, and then start the two BP neural network module, and finally the expert system gives fault diagnosis results and explain. For example, part processing rules is as follows(result is corresponding to the Fig.4): assign $x 1$ :=getnumber1(), ,...., if $\left(\mathrm{C}_{2} \mathrm{H}_{2} \mathrm{C}_{2} \mathrm{H}_{4}=0\right.$ and $\mathrm{CH}_{4} \mathrm{H}_{2}=2$ and $\mathrm{C}_{2} \mathrm{H}_{4} \mathrm{C}_{2} \mathrm{H}_{6}=1$ )(advice' Oil chromatographic analysis: $\mathrm{H}_{2}, \mathrm{CH}_{4}, \mathrm{C}_{2} \mathrm{H}_{6}, \mathrm{C}_{2} \mathrm{H}_{4}, \mathrm{C}_{2} \mathrm{H}_{2}, \mathrm{CO}, \mathrm{CO}_{2}$ ' $\mathrm{H}_{2}{ }^{\prime}$ ' $\mathrm{CH}_{4}{ }^{\prime}$ ' $\mathrm{C}_{2} \mathrm{H}_{6}$ ' ' $\mathrm{C}_{2} \mathrm{H}_{4}$ ' ' $\mathrm{C}_{2} \mathrm{H}_{2}$ ' ' $\mathrm{CO}$ ' ' $\mathrm{CO}_{2}$ \& ' Three ratio result: $\mathrm{C}_{2} \mathrm{H}_{2} / \mathrm{C}_{2} \mathrm{H}_{4} \mathrm{CH}_{4} / \mathrm{H}_{2} \mathrm{C}_{2} \mathrm{H}_{4} / \mathrm{C}_{2} \mathrm{H}_{6}$ ' $\mathrm{C}_{2} \mathrm{H}_{2} \mathrm{C}_{2} \mathrm{H}_{4}{ }^{\prime \prime} \mathrm{CH}_{4} \mathrm{H}_{2}{ }^{\prime}{ }^{\prime} \mathrm{C}_{2} \mathrm{H}_{4} \mathrm{C}_{2} \mathrm{H}_{6}{ }^{\prime}$, medium temperature overheat $\left(300^{\circ} \mathrm{C}-700^{\circ} \mathrm{C}\right)$. Possible fault cause: tap changer poor contact,iron core leakage flux, partial short-circuit.Treatment countermeasures: according to different cause of overheating fault,adopt different treatment strategies ( omitted ) .' \& ' ANN diagnosis result:' \& ' overheat: ' x1 \& ' arc discharge: ' x2 \& ' ANN ( characteristics gas ) diagnosis result:' \& ' general overheat fault:'xt1 \& ' serious overheat fault:' xt2 \& ' local discharge:'xt3 \& ' spark discharge:' xt4 \& ' arc discharge:'xt5) .

Adding some procedures or functions to the ESTA for implementing the BP neural network.By adding some procedures or functions to the ESTA, the research expert system can write/read data to/from some text files so as to realize data exchange of the expert system and the neural networks. For example, the definition of some procedures or functions added to the Veintf.pro of the ESTA are as follows:

savebyq5( $\left.\mathrm{X}_{1}, \mathrm{X}_{2}, \mathrm{X}_{3}, \mathrm{X}_{4}, \mathrm{X}_{5}\right)$; Providing the second $\mathrm{BP}$ artificial neural network with the input data. ann_tz_foresee (); $\quad$ Calling the second BP artificial neural network function module.

getnumber1(); $\quad$ Read data from a text file generated by the neural network.

proc_descr(savebyq5,5,[number,number,number,number,number]).

proc_descr(ann_tz_foresee, $0,[])$.

func_descr(getnumber1,0,[],number).

eval_proc(savebyq5,[P1,P2,P3,P4,P5]) :- number_real(P1,P11),number_real(P2,P22),

number_real(P3,P33),number_real(P4,P44),number_real(P5,P55), openwrite(auxfile2,

"d:II2014.1.12lltzforeseelldataforesee.txt"), writedevice(auxfile2), write(P11,' '), write(P22,'

'), write(P33,' '), write(P44,' '), write(P55,' '), closefile(auxfile2), !.

eval_func(getnumber1,[],float(P1)):-openread(auxfile2,"D:\|2014.1.12\|bpforeseel|result1.txt"),

readdevice(auxfile2), readln(L),str_real(L,P1),closefile(auxfile2),!.

eval_proc(ann_tz_foresee,[]) :- !, NullWin = cast $($ window, 0), IId=

vpi_CreateProcess(NullWin,"d:IV2014.1.12lltzforeseel|BPforesee.exe","",wsf_Restored),!.

\section{Discussions}

This paper uses a group of data(the part of values in Table3) came from the literature[8] to test the correctness of the DBN(see Fig.2), the three ratio, characteristic gas method,and the two BP artificial neural network model(see Fig.4). The test data of Table 4 come from the first 10 lines and the $\mathrm{H}_{2}$, $\mathrm{CO}, \mathrm{CH}_{4}, \mathrm{C}_{2} \mathrm{H}_{4}, \mathrm{C}_{2} \mathrm{H}_{2}, \mathrm{C}_{2} \mathrm{H}_{6}, \mathrm{CO}_{2}$ columns in Table 3. The Fig. 2 shows the calculation result of the DBN based on MATLAB.Table 4 shows the comparison of the computing result of the three ratio method, the first BP artificial neural network and the second. The test results are consistent with practical conclusion basically. These are some of the preliminary diagnosis, it still need to combine with other methods for integrated diagnosis. The paper discusses an integrated of the expert system based on the ESTA,the DBN and the BP artificial neural network programmed in MATLAB, and trying an application of the mixed deterministic reasoning combined with numerical calculation. Application shows that the operating efficiency is acceptable on a normal PC.

\section{Conclusions}

1) Some procedures or functions had be added to the ESTA so that the ESTA function was extended and the ESTA can communicate with MATLAB via some data files. 
2) The DBN based on DGA and MATLAB was created. The DBN based on DGA was explored in the application of transformer fault diagnosis by the training and testing data sets , and the testing also shows that the DBN has feasibility and effectiveness in the transformer fault diagnosis.

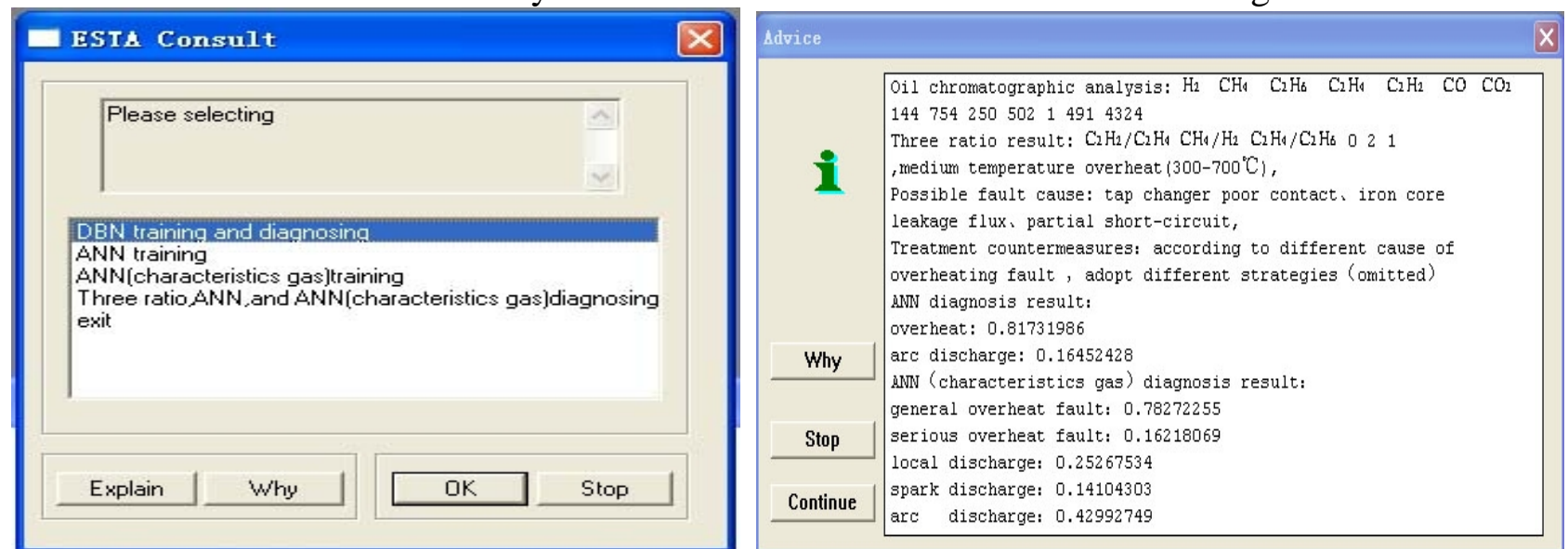

Fig. 3 choice interface of the training and diagnosing Fig.4 result of the three ratio and BP diagnosis

Table 4 the comparison of the computing result of the three fault diagnosis model

\begin{tabular}{|c|l|l|l|}
\hline Data No. & \multicolumn{1}{|c|}{ The three ratio method } & The first ANN & The second ANN \\
\hline 1 & high temperature overheat & arc discharge & serious overheat \\
\hline 2 & medium temperature overheat & overheat & general overheat \\
\hline 3 & arc discharge and overheat & arc discharge & serious overheat \\
\hline 4 & high temperature overheat & overheat & serious overheat or arc discharge \\
\hline 5 & low energy (spark)discharge & arc discharge & arc discharge \\
\hline 6 & high temperature overheat & overheat & serious overheat \\
\hline 7 & arc discharge & overheat & serious overheat or arc discharge \\
\hline 8 & low temperature overheat & overheat & general overheat \\
\hline 9 & high temperature overheat & overheat & general overheat \\
\hline 10 & medium temperature overheat & arc discharge & serious overheat \\
\hline
\end{tabular}

3) Two artificial neural network based on BP programmed in MATLAB were given by using the directly DGA method and the characteristic gas based on fuzzy processing in transformer oil, which mades it possible to train, diagnose, predict and visualize.

4) The transformer fault diagnosis expert system based on the $\mathrm{DBN}$, the three ratio and characteristic gas method of DGA including the two BP artificial neural network had be created via the ESTA and MATLAB. The basic application shows the effectiveness of the expert system. The four diagnostic models complement each other, which increases reliability of the diagnosis result.

Because of the training samples is small, it is necessary to further working in order to improve the accuracy of diagnosis and to expand the scope of it. Exploring the combination application of the DBN, expert system (such as based on the ESTA) and MATLAB has certain practical significance for establishing some assistance decision support systems.

\section{References}

[1] Xu Yuanfang,Zhou Yang, Zheng Hua. Implementation research of BP neural network based on MATLAB. Micro computer Applications[J]. 2006,22(8) : 41-44.

[2] Zhao Minhao. Data Fusion Based on Deep Learning and Its Application for FPSO Monitoring System[D].TianJin: TianJin University (2013) (In Chinese)

[3] Bian Li, Bian Chenyuan. Review on intelligence fault diagnosis in power networks, Power System Protection and Control [J].2014,42(3):146-153.

[4] Wu Jinghong,Zhu Zhuhui. Development and application of fault detection system for power transformer. Zhejiang Hydrotechnics[J], 2001,4:62-64.

[5] Wan Yiqin. Transformer fault diagnosis based on probabilistic neural network. Master's degree thesis, 2007. 
[6] Prolog Development Center.Esta document. http://www.Visual-Prolog.com,2001[EB/OL].

[7] Shi Feng,Wang Xiaochuan,Yu Lei,Li Yang. MATLAB neural network 30 case analysis[M], Beihang university press, 2009. 